This item was submitted to Loughborough's Research Repository by the author.

Items in Figshare are protected by copyright, with all rights reserved, unless otherwise indicated.

\title{
Bi-stable tunneling current through a molecular quantum dot
}

PLEASE CITE THE PUBLISHED VERSION

LICENCE

CC BY-NC-ND 4.0

\section{REPOSITORY RECORD}

Alexandrov, A.S., A.M. Bratkovsky, and R.S. Williams. 2019. "Bi-stable Tunneling Current Through a Molecular Quantum Dot". figshare. https://hdl.handle.net/2134/1160. 
This item was submitted to Loughborough's Institutional Repository (https://dspace.lboro.ac.uk/) by the author and is made available under the following Creative Commons Licence conditions.

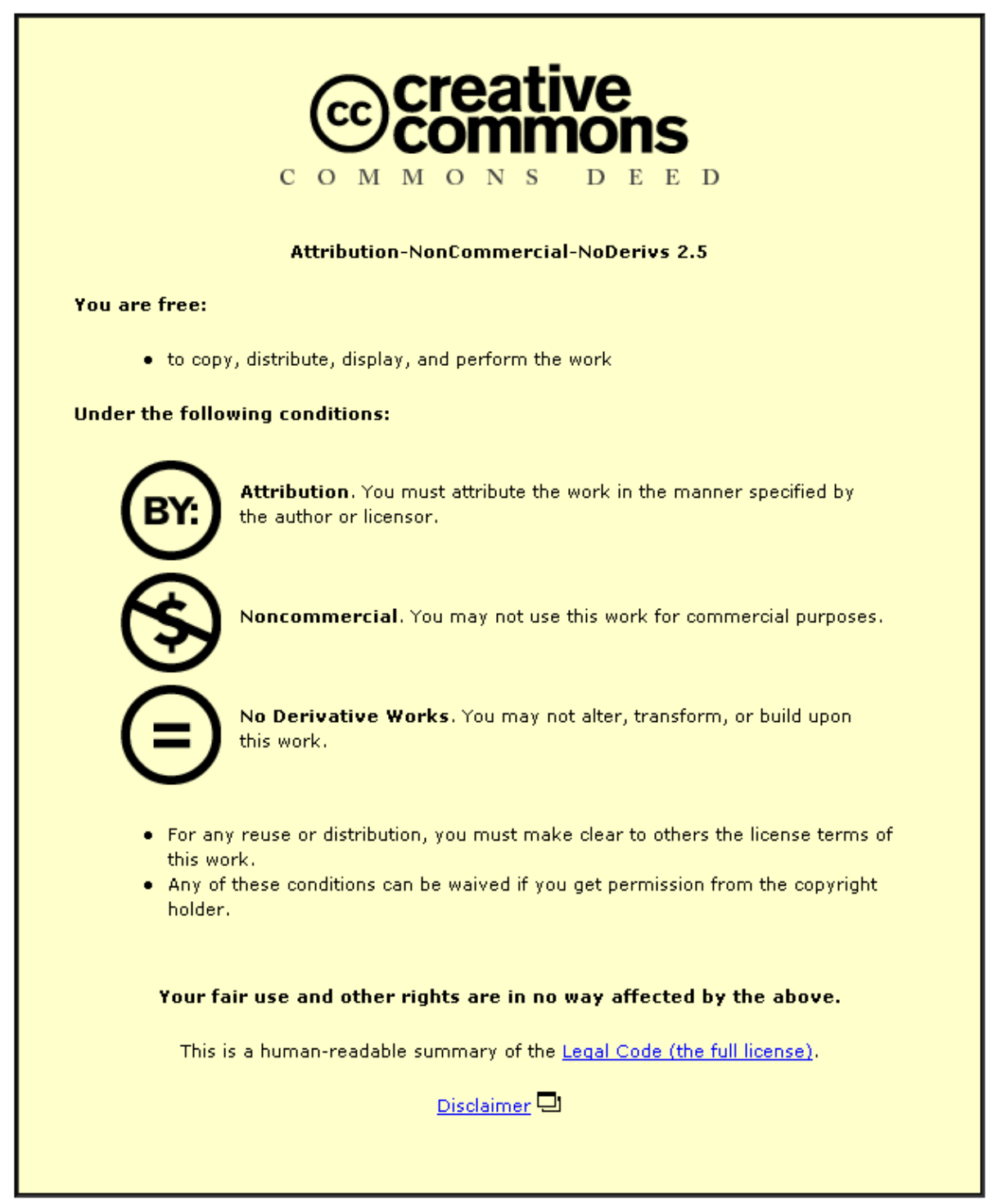

For the full text of this licence, please go to: http://creativecommons.org/licenses/by-nc-nd/2.5/ 


\title{
Bi-stable tunneling current through a molecular quantum dot
}

\author{
A.S. Alexandrov ${ }^{1,2}$, A.M. Bratkovsky ${ }^{1}$, and R. Stanley Williams ${ }^{1}$ \\ ${ }^{1}$ Hewlett-Packard Laboratories, 1501 Page Mill Road, 1L, Palo Alto, California 94304 \\ ${ }^{2}$ Department of Physics, Loughborough University, Loughborough LE11 3TU, United Kingdom
}

(April 17, 2002)

\begin{abstract}
An exact solution is presented for tunneling through a negative- $U$ degenerate molecular quantum dot weakly coupled to electrical leads. The tunnel current exhibits hysteresis if the level degeneracy of the negative- $U$ dot is larger than two. Switching occurs in the voltage range $V_{1}<V<V_{2}$ as a result of attractive electron correlations in the molecule, which open up a new conducting channel when the voltage is above the threshold bias voltage $V_{2}$. Once this current has been established, the extra channel remains open as the voltage is reduced down to the lower threshold voltage $V_{1}$. Possible realizations of bi-stable molecular quantum dots are fullerenes, especially $C_{60}$, and mixed-valence compounds.
\end{abstract}

PACS: 85.65.+h, 73.63.-b,73.63.Nm, 71.38.Mx

Molecular-scale electronics is currently a very active area of research [1]. The present goals for this field are to design and characterize molecules that could be the "transmission lines" [2,3] and active elements in electronic circuitry 11, 1 . The dominant mechanism of transport through active devices will most likely be resonant tunneling through electronic molecular states 4 (see also [5.6\% and references therein). A few experimental studies [4, 7] provide evidence for various molecular switching effects, where the current-voltage (I-V) characteristics show two branches with high and low current for the same voltage. This remarkable phenomenon can result from a conformational transformation of certain molecules containing a "moving part" like a bypyridinium ring, which changes its position if the voltage is sufficiently high. The transformation necessarily involves a large displacement of many atoms so that this ionic switching is rather slow, perhaps operating on a millisecond scale. Switching has also been observed for simple molecules (organic molecular films), and in some cases is strongly dependent on the choice of contacts and substrates [7]. Molecular devices that exhibit bi-stability and fast switching could be the basis of future oscillators, amplifiers and other important circuit elements. Thus, further progress in molecular electronics will depend upon finding molecules and understanding intrinsic mechanisms for their reversible switching from low- to high-current states.

In this Letter, we study a model quantum dot, which exhibits an intrinsic electronic switching of the current state due to attractive electron correlations. We show that if the degeneracy is larger than two, the tunnel current becomes bistable in some voltage range and the dot exhibits a current hysteresis as a function of bias voltage. In the simplest case of a doubly degenerate level, the bistability does not occur. We present the exact solution of the model, allowing for a detailed analysis of the current bistability.

Repulsive electron correlations cause the "Coulomb blockade" in the I-V characteristics of quantum dots [8]. However, they cannot cause any switching. Here, we show that a negative Hubbard $U$ of any origin can provide an intrinsic non-retarded current switching of a molecular quantum dot. One mechanism, that can produce a negative $U$ in molecular systems, is a strong electronphonon (vibronic) interaction. If the tunneling time is comparable to or larger than the characteristic phonon times, a polaron is formed inside the molecular wire [9]. There is a wide range of bulk molecular conductors with polaronic carriers. Since the formation of polarons in polyacetylene (PA) was theoretically discussed [10], they were detected optically in PA [11], in conjugated polymers such as polyphenylene, polypyrrole, polythiophene, polyphenylene sulfide [12], Cs-doped biphenyl [13], ndoped bithiophene [14, polyphenylenevinylene(PPV)based light emitting diodes [15], and other molecular systems. In contrast to bare electrons, polarons attract each other at short distances of the order of the interatomic spacing and form small bipolarons [16. Bipolaron formation can strongly affect the transport properties of long molecular wires, as discussed recently [17]. When bipolarons are not formed in molecular quantum dots because of the short life-time of the carrier inside the molecule, the attractive correlations between carriers still remain. Moreover, attractive short-range correlations (negative Hubbard $U$ ) are feasible even without electron-phonon interactions. For example, they might be of a pure "chemical" origin, as in the mixed valence complexes [18].

Our starting point is the tunneling Hamiltonian, which includes a negative Hubbard $U$ in the molecular eigenstate $\varepsilon_{\mu}$ coupled with the left and right leads by the hopping integrals $h_{\alpha k \mu}$

$$
H=\sum_{\mu} \varepsilon_{\mu} \hat{n}_{\mu}+\frac{1}{2} U \sum_{\mu \neq \mu^{\prime}} \hat{n}_{\mu} \hat{n}_{\mu^{\prime}}+\sum_{k, \alpha} \xi_{\alpha k} a_{\alpha k}^{\dagger} a_{\alpha k}
$$




$$
+\sum_{k, \mu, \alpha}\left(h_{\alpha k \mu} a_{\alpha k}^{\dagger} c_{\mu}+\text { H.c. }\right) .
$$

Here $a_{\alpha k}$ and $c_{\mu}$ are the annihilation operators in the left $(\alpha=1)$ and right $(\alpha=2)$ leads, and in the molecule, respectively, $\hat{n}_{\mu}=c_{\mu}^{\dagger} c_{\mu}, \xi_{\alpha k}$ is the energy dispersion in the leads, and $U<0$. This negative $U$ Hamiltonian is derived from the general microscopic Hamiltonian with the bare electron-phonon (vibron) interaction and Coulomb repulsion using the canonical transformation 16. It was successfully applied to glassy semiconductors 19,20], high $T_{c}$ superconductors [16,21] including doped fullerenes [22] mixed valence compounds [18], and more recently to superconductor-insulator-superconductor tunneling [23. The current through the molecular quantum dot is conveniently expressed in terms of the molecular density of states (DOS) $\rho_{\mu}(\omega)$ as 8

$$
I=e \int_{-\infty}^{\infty} d \omega\left[f_{1}(\omega)-f_{2}(\omega)\right] \sum_{\mu} \Gamma_{\mu}(\omega) \rho_{\mu}(\omega),
$$

where $f_{1,2}(\omega)=\{\exp [(\omega+\Delta \mp e V / 2) / T]+1\}^{-1}$, $\Delta$ is the position of the lowest unoccupied molecular level with respect to the chemical potential, $\Gamma_{\mu}(\omega)=$ $\left.\Gamma_{1 \mu}(\omega) \Gamma_{2 \mu}(\omega) /\left[\Gamma_{1 \mu}(\omega)\right)+\Gamma_{2 \mu}(\omega)\right]$, and $\Gamma_{\alpha \mu}(\omega)=$ $2 \pi \sum_{\alpha k}\left|h_{\alpha k \mu}\right|^{2} \delta\left(\omega-\xi_{\alpha k}\right)$. Molecular DOS is given by $\rho_{\mu}(\omega)=-(1 / \pi) \operatorname{Im} G_{\mu}^{(1)}(\omega)$, where $G_{\mu}^{(r)}(\omega)$ is the Fourier transform of the $r$-particle retarded Green's function (GF) defined as

$$
G_{\mu}^{(r)}(t)=-i \theta(t) \sum_{\mu_{1} \neq \mu_{2} \neq \ldots \mu}\left\langle\left\{c_{\mu}(t) \prod_{i=1}^{r-1} \hat{n}_{\mu_{i}}(t), c_{\mu}^{\dagger}\right\}\right\rangle
$$

for $2 \leq r<\infty$ and $G_{\mu}^{(1)}(t)=-i \theta(t)\left\langle\left\{c_{\mu}(t), c_{\mu}^{\dagger}\right\}\right\rangle$. Here $\{\ldots, \ldots\}$ is the anticommutator, and $\theta(t)=1$ for $t>0$ and zero otherwise. In the following, we apply perturbation theory with respect to the hopping integrals, neglecting any contribution to the current other than $h^{2}$, but keeping all orders of the negative Hubbard $U$. Terms of higher order in $h$ cannot change the gross I-V features for any voltage except the narrow transition regime from one lead to another. Then, applying the equations of motion for the Heisenberg operators $c_{\mu}(t), \hat{n}_{\mu}(t)$ and $a_{\alpha k}(t)$, we obtain an infinite set of coupled equations for the molecular GFs as

$$
\begin{aligned}
i \frac{d G_{\mu}^{(r)}(t)}{d t}= & \delta(t) \sum_{\mu_{1} \neq \mu_{2} \neq \ldots \mu} \prod_{i=1}^{r-1} n_{\mu_{i}}(0)+ \\
& {\left[\varepsilon_{\mu}+(r-1) U\right] G_{\mu}^{(r)}(t)+U G_{\mu}^{(r+1)}(t), }
\end{aligned}
$$

where $n_{\mu}(t)=\left\langle c_{\mu}^{\dagger}(t) c_{\mu}(t)\right\rangle$ is the expectation number of electrons on the conducting molecular level. For the sake of analytical transparency we solve this system for a molecule having one $d$-fold degenerate energy level with $\varepsilon_{\mu}=0$. In this case the set appears to be finite, and it can be solved by using the Fourier transformation. Fourier transforming Eqs.(4) we find the one-particle GF as

$$
G_{\mu}^{(1)}(\omega)=\sum_{r=0}^{d-1} \frac{Z_{r}(n)}{\omega-r U+i \delta}
$$

where $\delta=+0, n=n_{\mu}(0)$, and

$$
Z_{r}(n)=\frac{(d-1) !}{r !(d-1-r) !} n^{r}(1-n)^{d-1-r} .
$$

This is an exact solution with respect to correlations which satisfies all sum rules. The electron density $n_{\mu}(t)$ obeys the rate equation, which is obtained by using the equations of motion as

$$
\frac{d n_{\mu}(t)}{d t}=2 \sum_{\alpha, k} h_{\alpha k \mu} \operatorname{Im} A_{\alpha k \mu}^{(1)}(t),
$$

where

$$
A_{\alpha k \mu}^{(r)}(t)=\sum_{\mu_{1} \neq \mu_{2} \neq \ldots \mu}\left\langle c_{\mu}^{\dagger}(t) \prod_{i=1}^{r-1} \hat{n}_{\mu_{i}}(t) a_{\alpha k}(t)\right\rangle .
$$

These correlation functions should be calculated to first order with respect to the hopping integrals $h$. In this order, they satisfy the infinite set of coupled equations

$$
\begin{aligned}
i \frac{d A_{\alpha k \mu}^{(r)}(t)}{d t}= & h_{\alpha k \mu}\left[n_{\mu}(t)-f\left(\xi_{\alpha k}\right)\right] \sum_{\mu_{2} \neq \ldots \mu} \prod_{i=1}^{r-1} n_{\mu_{i}}(t)+ \\
& U A_{\alpha k \mu}^{(r+1)}(t)+\left[\xi_{\alpha k}-(r-1) U\right] A_{\alpha k \mu}^{(r)}(t) .
\end{aligned}
$$

But if we have a finite number of molecular states, the set is finite like Eqs.(4). One readily solves this set in the steady state, when $n_{\mu_{i}}(t)$ and $A_{\alpha k \mu}^{(r)}(t)$ become timeindependent. For a $d$-fold degenerate energy level, the one-particle correlation function is found as

$$
A_{\alpha k \mu}^{(1)}=\left[n-f\left(\xi_{\alpha k}\right)\right] h_{\alpha k \mu} \sum_{r=0}^{d-1} \frac{Z_{r}(n)}{\xi_{\alpha k}-r U+i \delta} .
$$

Substituting Eq. (9) into Eq. (6), we obtain the steady state equation for the electron density on the molecule as

$$
\sum_{\alpha} \sum_{r=0}^{d-1} \Gamma_{\alpha}(r U)\left[n-f_{\alpha}(r U)\right] Z_{r}(n)=0 .
$$

Here we assume that $\Gamma_{\alpha \mu}(\omega)=\Gamma_{\alpha}(\omega)$ does not depend on $\mu$, otherwise the degeneracy would be removed. To simplify the mathematics further, we now assume that $\Gamma_{\alpha}(\omega)=\Gamma$ is a constant. Then, from Eq. (2) and Eq. (5) the current is found as

$$
j=\sum_{r=0}^{d-1}\left[f_{1}(r U)-f_{2}(r U)\right] Z_{r}(n),
$$


where $j=I / I_{0}$ with $I_{0}=e d \Gamma / 2$. Let us consider twofold, four-fold, and six-fold degenerate molecular level. For $d=2$ the kinetic equation is linear in $n$, and there is only one solution,

$$
n=\frac{\sum_{\alpha} f_{\alpha}(0)}{2+\sum_{\alpha}\left[f_{\alpha}(0)-f_{\alpha}(U)\right]} .
$$

The current through a two-fold degenerate molecular dot is found as

$$
j=2 \frac{f_{1}(0)\left[1-f_{2}(U)\right]-f_{2}(0)\left[1-f_{1}(U)\right]}{2+\sum_{\alpha}\left[f_{\alpha}(0)-f_{\alpha}(U)\right]} .
$$

There is no current bistability in this case. Moreover, if the temperature is low $(T \ll \Delta,|U|)$ there is practically no effect of correlations on the current, $j \approx V \theta(e|V|-$ $2 \Delta) /|V|$.

Remarkably, four-fold or higher- degenerate negative $U$ dots reveal a switching effect. In this case, the kinetic equation is nonlinear, allowing for a few solutions. If $\mathrm{eV}<2(\Delta-|U|)$, the only physically allowed solution of Eq.(10) for $d=4$ and $d=6$ at zero temperature is $n=0$. If $2(\Delta-|U|)<e V<2 \Delta, T=0$ and $|U|<2 \Delta / d$, the kinetic equation is reduced to

$$
2 n=1-(1-n)^{d-1} .
$$

For $d=4$ it has two physical roots, $n=0$ and $n=$ $\left(3-5^{1 / 2}\right) / 2 \approx 0.38$ 24. In this voltage range $f_{1}(0)=$ $f_{2}(r U)=0$, but $f_{1}(U)=f_{1}(2 U)=f_{1}(3 U)=1$ at $T=0$, when $|U|<\Delta / 2$. Using the sum rule $\sum_{r=0}^{d-1} Z_{r}(n)=1$ and the kinetic equation (10), the current is simplified in this voltage range as $j=2 n$. Hence we obtain two stationary states of the molecule with low (zero at $T=0$ ) and high current, $I \approx 0.76 I_{0}$ for the same voltage in the range $2(\Delta-|U|)<e V<2 \Delta$. For $d=6$, the kinetic equation has two physical roots in this voltage range, $n=0$ and $n \approx 0.48$, which corresponds to $I=0$ and $I \approx 0.96 I_{0}$, respectively. Above the standard threshold, $e V>2 \Delta$, where $f_{1}(r U)=1$ and $f_{2}(r U)=0$, when $|U|<\Delta / 3$, there is only one solution, $n=0.5$ with the current $I=I_{0}$.

One can better understand the origin of the switching phenomenon by taking the limit $d \gg 1$. The physical roots of Eq. (14) are $n=0$ and $n=0.5$ in this limit with the current $I=0$ and $I=I_{0}$, respectively. This is precisely the solution of the problem in the mean-field approximation (MFA), which is a reasonable approximation for $d \gg 1$. Indeed, using MFA one replaces the exact two-body interaction in the Hamiltonian for a mean-field potential as $\frac{1}{2} U \sum_{\mu \neq \mu^{\prime}} \hat{n}_{\mu} \hat{n}_{\mu^{\prime}} \approx$ $U \sum_{\mu \neq \mu^{\prime}} \hat{n}_{\mu} n_{\mu^{\prime}}-\frac{1}{2} U \sum_{\mu \neq \mu^{\prime}} n_{\mu} n_{\mu^{\prime}}$. Then the MFA DOS is given by $\rho_{\mu}(\omega)=\delta[\omega-U(d-1) n]$. Using the FermiDirac Golden rule the rate equation for $n$ becomes

$$
\frac{d n}{d t}=-2 \Gamma n+\Gamma \sum_{\alpha} f_{\alpha}[n U(d-1)] .
$$

For $T=0$ there are two stationary solutions of Eq. (15), $n=0$ and $n=0.5$ in the voltage range $2(\Delta-|\tilde{U}|)<$ $\mathrm{eV}<2 \Delta$, and only one solution, $n=0.5$ for $e V>2 \Delta$, where $\tilde{U}=U(d-1) / 2$. The MFA current is found as

$$
j=f_{1}(2 n \tilde{U})-f_{2}(2 n \tilde{U}) .
$$

Combining this equation and the rate equation (15) with $d n / d t=0$, we obtain the I-V characteristic equation as

$$
\begin{aligned}
& \frac{|\tilde{U}|}{\Delta}(1-R)=1-\frac{T}{\Delta} \\
& \times \ln \left[\frac{(1+R) \sinh (e V / 2 T)}{j}-\cosh (e V / 2 T)\right],
\end{aligned}
$$

where

$$
R=\left\{[1-j \operatorname{coth}(e V / 2 T)]^{1 / 2}-\frac{j^{2}}{\sinh ^{2}(e V / 2 T)}\right\}^{1 / 2} .
$$

The $I / V$ curves are shown in Fig. 1 for different temperatures and $|\tilde{U}|=0.9 \Delta$. Interestingly, the temperature narrows the voltage range of the hysteresis loop, but the transition from the low (high)-current branch to the high (low)-current branch remains discontinuous.

Let us examine the stability of each branch in the framework of the MFA rate equation (15). Introducing small fluctuations of the electron density as $n(t)=$ $n+\delta n \exp (\gamma t)$ and linearizing Eq.(15) with respect to $\delta n$ we find the increment $\gamma$,

$$
\begin{aligned}
\gamma= & -2 \Gamma+\frac{|\tilde{U}| \Gamma}{2 T} \cosh ^{-2}\left(\frac{\Delta-2 n|\tilde{U}|-e V / 2}{2 T}\right) \\
& +\frac{|\tilde{U}| \Gamma}{2 T} \cosh ^{-2}\left(\frac{\Delta-2 n|\tilde{U}|+e V / 2}{2 T}\right) .
\end{aligned}
$$

One can see from this equation that at temperatures $T \ll$ $|\tilde{U}|$ the low-current branch $(n=0)$ looses its stability at the threshold $V_{2}=2 \Delta / e$, while the high-current branch looses its stability at $V_{1}=2(\Delta-|\tilde{U}|) / e$. In the voltage range $V_{1}<V<V_{2}$ both branches are stable, $\gamma \approx-2 \Gamma<$ 0 .

Finally, let us analyze the effect of a splitting of the degenerate molecular level on the bi-stability. The degeneracy could be removed because of Jahn-Teller distortions and/or the coupling with the leads. We assume that $d \gg 1$ levels are evenly distributed in a band of a width $W$. Then the MFA rate equation (15) is modified as

$$
\frac{d n_{\mu}}{d t}=-2 \Gamma n_{\mu}+\Gamma \sum_{\alpha} f_{\alpha}\left(\varepsilon_{\mu}+N U\right),
$$

where $N=\sum_{\mu^{\prime}} n_{\mu^{\prime}}$. For $T=0$ in the stationary regime $\left(d n_{\mu} / d t=0\right)$ it has two solutions, $N=0$ and $N=d / 2$, 


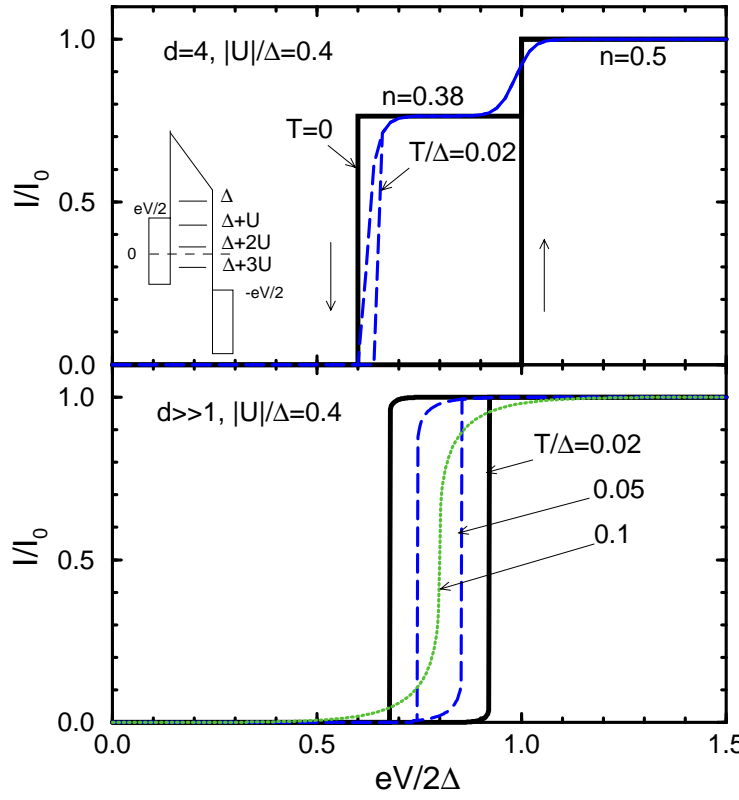

FIG. 1. The current-voltage hysteresis loop for the negative- $U$ molecular quantum dot for the molecular level with the degeneracies $d=4$ and $d \gg 1$. The two cases are very different in some aspects. Top panel: $d=4$, $|U| / \Delta=0.4$. In the hysteretic region the current jumps to the value $I / I_{0}=0.76$ at the lower threshold $\left(e V_{1} / 2 \Delta=0.6\right)$ and then to the value $I / I_{0}=1$ at the higher threshold $\left(e V_{2} / 2 \Delta=1\right)$. Bottom panel: highly degenerate molecular level with $d \gg 1,|\tilde{U}| / \Delta=0.4$. The current jumps directly to the value $I / I_{0}=1$ at the lower threshold. Temperature quickly reduces the width of the hysteretic region, much faster for $d=4$ than for $d \gg 1$. In the latter case the hysteresis is over at about $T / \Delta=0.1$. Inset in the top panel shows a schematic of the energy level diagram for $d=4$ quantum dot under bias voltage $V$.

in the voltage range $V_{1}+W<V<V_{2}$ with the current $j=0$ and $j=1$, respectively. We conclude that the level splitting $W<|U|$ leads to a narrowing of the voltage range of the bi-stability similar to the temperature narrowing shown in Fig.1. A parameter-free estimate of the negative Hubbard $U$ in some oxides yields $|U|$ about a few tens of eV [25]. We expect a negative $U$ of the same order of magnitude in carbon-based compounds. Among potential candidates for the negative $U$ quantum dot are a single $C_{60}$ molecule $(d=6)$, where the electron-vibronic coupling proved to be particularly strong [26], or other fullerenes including short nanotubes $(d \gg 1)$ connected to metal electrodes. Other likely candidates are mixedvalence molecular complexes [18]. There should be no retardation of the switching on the time scale above the inverse vibron (phonon) frequency, which is $10^{-14} \mathrm{~s}$ or less in carbon-based compounds.

In conclusion, we have introduced and solved a model for tunneling through a negative- $U$ degenerate molecular dot weakly coupled to electrical leads. The exact many-particle Green's functions of a $d$-fold degenerate molecular level, the density of states and the nonlinear rate equation for the electron density on the molecule have been derived. We have found the exact solutions for the carrier population in the dot and the current for the degeneracies of the molecular level $d=2,4,6$, and $d \gg 1$. The current-voltage characteristics show a hysteretic behavior for $d>2$ over a finite voltage range. When the voltage increases from zero, the molecule remains in a low-current state until the threshold $V_{2}$ is reached. Remarkably, when the voltage decreases from the value above the threshold $2 \Delta / e$, the molecule remains in the high-current state down to the voltage $V_{1}=(2 \Delta-|U|) / e$, well below the threshold $V_{2}$. This mechanism for electronic molecular switching without retardation requires many-particle attractive correlations, which can arise from strong electron-vibronic coupling and/or mixed valence states. Experimental verification of such bi-stable systems will require careful collection and analysis of both forward and reverse voltage sweeps of the tunneling current through candidate molecules. The forward voltage sweep by itself will resemble a standard Coulomb blockade I-V characteristic with a turn-on voltage of $V_{2}$, whereas the reverse sweep should reveal hysteresis.

This work has been partly supported by DARPA. The authors acknowledge useful discussions with P. Kornilovich and D. Stewart.

[1] A. Aviram and M. Ratner, Eds., Molecular Electronics: Science and Technology (Ann. N.Y. Acad. Sci., New York, 1998).

[2] J. M. Lehn, Angew. Chem. Int. Ed. Engl. 29, 1304 (1990).

[3] J. M. Tour, Acc. Chem. Res. 33, 791 (2000); J. M. Tour et al., J. Am. Chem. Soc. 117, 9529 (1995).

[4] C. P. Collier et al., Science 285, 391 (1999); J. Chen et al., Science 286, 1550 (1999); D. I. Gittins et al., Nature (London) 408, 677 (2000).

[5] A. Aviram and M. A. Ratner, Chem. Phys. Lett. 29, 257 (1974); S. Datta et al., Phys. Rev. Lett. 79, 2530 (1997).

[6] P. E. Kornilovitch and A. M. Bratkovsky, Phys. Rev. B 64, 195413 (2001).

[7] D. Stewart, Y. Chen and R.S. Williams, unpublished.

[8] N. S. Wingreen and Y. Meir, Phys. Rev. B 49, 11040 (1994) and references therein.

[9] H. Ness, S. A. Shevlin, and A. J. Fisher, Phys. Rev. B 63, 125422 (2001).

[10] W. P. Su and J. R. Schrieffer, Proc. Natl. Acad. Sci. 77, 5626 (1980).

[11] A. Feldblum et al., Phys. Rev. B 26, 815 (1982).

[12] R. R. Chance, J. L. Bredas, and R. Silbey, Phys. Rev. B 29, 4491 (1984).

[13] M. G. Ramsey et al., Phys. Rev. B 42, 5902 (1990). 
[14] D. Steinmuller, M. G. Ramsey, and F. P. Netzer, Phys. Rev. B 47, 13323 (1993).

[15] L. S. Swanson et al., Synth. Metals 55, 241 (1993).

[16] A. S. Alexandrov and N. F. Mott, Polarons and Bipolarons (World Scientific, Singapore, 1995).

[17] A. S. Alexandrov, A.M. Bratkovsky and P.E. Kornilovitch, Phys. Rev. B 65, 155209 (2002).

[18] J. A. Wilson, J. Phys.-Cond. Matter 13, R945 ( 2001).

[19] P.W. Anderson, Phys. Rev. Lett. 34, 953 (1975).

[20] R.A. Street and N.F. Mott, Phys. Rev. Lett. 35, 1293 (1975).

[21] R. Micnas, J. Ranninger, and S. Robaszkiewicz, Rev Mod Phys 62, 113 (1990), and references therein.

[22] A.S. Alexandrov, V.V. Kabanov, Phys. Rev. B54, 3655 (1996).

[23] V. Oganesyan, S. Kivelson, T. Geballe, and B. Moyzhes, Phys. Rev. B 65, 172504 (2002).

[24] Recently V. N. Ermakov (Physica E 8, 99 (2000)) calculated the I-V curves of a four-fold degenerate dot with the Coulomb and electron-phonon interactions. He observed a switching effect in the numerical I-V curves, similar to that in the negative- $U$ Hubbard model discussed here. However, he obtained an unphysical population of each molecular state, $n=1$, different from our $n=0.38$ for the initial step and $n=0.5$ for all the biases above the upper threshold voltage.

[25] A. S. Alexandrov and A. M. Bratkovsky, Phys. Rev. Lett. 84, 2043 (2000).

[26] H. Park, J. Park, A.K.L. Lim, E.H. Anderson, A.P. Alivisatos, P.L. McEuen, Nature 407, 57 (2000). 